\title{
Estrogen-dependent effects of 5-hydroxytryptophan on cortical spreading depression in rat: Modelling the serotonin-ovarian hormone interaction in migraine aura
}

\author{
Virginie Chauvel ${ }^{1}$, Sylvie Multon ${ }^{1 *}$ and Jean Schoenen ${ }^{1,2 *}$ \\ ${ }^{1}$ Cephalic Pain Unit of GIGA-Neurosciences, Liège University, Liège, Belgium \\ ${ }^{2}$ Headache Research Unit, Department of Neurology, Liège University, Citadelle Hospital, Liège, \\ Belgium \\ ${ }^{*}$ These authors have contributed equally to this work.
}

\begin{abstract}
Background: Cortical spreading depression (CSD) is the likely culprit of the migraine aura. Migraine is sexually dimorphic and thought to be a "low $5-\mathrm{HT}$ " condition. We sought to decipher the interrelation between serotonin, ovarian hormones and cortical excitability in a model of migraine aura.

Methods: Occipital $\mathrm{KCl}$-induced CSDs were recorded for one hour at parieto-occipital and frontal levels in adult male $(n=16)$ and female rats $(n=64)$ one hour after intraperitoneal (i.p.) injection of 5hydroxytryptophan (5-HTP) or $\mathrm{NaCl}$.

Sixty-five oophorectomized females were treated with estradiol- (E2) or cholesterol- (Chol) filled capsules. Two weeks later we recorded CSDs after $5-\mathrm{HTP} / \mathrm{NaCl}$ injections before or 20 hours after capsule removal.

Results: 5-HTP had no effect in males, but decreased CSD frequency in cycling females, significantly so during estrus, at parieto-occipital $(-3.5 \mathrm{CSD} / \mathrm{h}, p<0.001)$ and frontal levels $(-2.5 \mathrm{CSD} / \mathrm{h}, p=0.014)$. In oophorectomized rats, CSD susceptibility increased during E2 treatment at both recording sites $(+5 \mathrm{CSD} / \mathrm{h}, p=0.001$ and $+3 \mathrm{CSD} / \mathrm{h}, p<0.01)$, but decreased promptly after E2 withdrawal ($4.7 \mathrm{CSD} / \mathrm{h}, p<0.001$ and $-1.7 \mathrm{CSD} / \mathrm{h}, p=0.094)$. The CSD inhibitory effect of 5 -HTP was significant only in E2-treated rats $(-3.4 \mathrm{CSD} / \mathrm{h}, p=0.006$ and $-1.8 \mathrm{CSD} / \mathrm{h}, p=0.029)$. Neither the estrous cycle phase, nor E2 or 5-HTP treatments significantly modified CSD propagation velocity.
\end{abstract}

Conclusion: 5-HTP decreases CSD occurrence in the presence of ovarian hormones, suggesting its potential efficacy in migraine with aura prophylaxis in females. Elevated E2 levels increase CSD susceptibility, while estrogen withdrawal decreases CSD. In a translational perspective, these findings may explain why migraine auras can appear during pregnancy and why menstrual-related migraine attacks are rarely associated with an aura.

\section{Keywords}

Cortical spreading depression, migraine with aura, serotonin, estrogen, 5-hydroxytryptophan 


\section{Introduction}

Migraine is the most common neurological disorder, affecting on average more than $15-20 \%$ of the general population with a female/male ratio of $3 / 1$ (1). Gonadal steroids determine the marked sexual dimorphism, especially estrogen (1). In $20-30 \%$ of patients, the migraine headache is preceded or accompanied by transient neurological symptoms called aura. There is strong evidence that the migraine aura is due to cortical spreading depression (CSD) (2), i.e. a slowly progressing wave (3-5 $\mathrm{mm} / \mathrm{min}$ ) of brief neurono-glial depolarization followed by a long-lasting suppression of neuronal activity and excitability (3). In animals, CSD can activate second order trigeminovascular nocicep-tors in the trigemino-cervical complex (4). In migraine with aura, CSD-induced trigeminovascular activation may be responsible for the migrainous headache, but not all auras are associated with headache (5).

There is evidence that ovarian hormones can influence CSD susceptibility. CSD thresholds are lower in female than in male mice (6). In neocortical slices, female sex hormones increase CSD occurrence and amplitude (7). We have previously shown that sexual hormones modulate the suppressive effect of systemic L-kynurenine on CSD over the estrous cycle (8) and in oophorectomized rats (9). The increased susceptibility to CSD of female FHM1 knock-in mice is abolished by oophorectomy and partially restored by estradiol treatment (10). Furthermore, in contrast to attacks of migraine without aura, which can be triggered by the premenstrual fall of plasma estradiol levels and tend to disappear during pregnancy, migraine with aura is rather favored by persistent high levels of female hormones, such as during pregnancy (11) or treatment with estrogen-containing drugs (12).

Migraine is considered as a "low serotonin" condition (13). Serotonin (5-HT) is synthesized from tryptophan, which is transformed into 5-hydroxytryptophan (5-HTP) via the rate limiting enzyme tryptophan hydroxylase (TPH). 5-HTP is then decarboxylated by the aromatic L-amino acid decarboxylase to form $5-\mathrm{HT}$. 5-HT is catabolized by monoamine oxidase into 5-hydroxyindolacetic (5-HIAA). Between attacks, migraineurs have low 5-HT and high 5-HIAA plasma levels, whereas the opposite is found during the attack (14). The altered levels of 5-HT and its metabolite found in plasma and urine (15) are thought to reflect dysfunctions occurring not only in blood platelets, but also in the brain (16), which is supported by the increase in cerebrospinal fluid 5-HIAA levels in migraine patients (14). A positron emission tomography study found that brain tryptophan uptake was reduced between attacks in migraine patients compared to controls, but increased during an attack (17).

In animals, 5-HT depletion by injection of para-chlorophenylalanine, a TPH inhibitor, enhances CSDinduced trigeminal nociception by increasing both CSD susceptibility and sensitivity of the trigeminal nocicep-tive system (18). Interictal low 5-HT transmission and its ictal increase may be responsible respectively for the abnormal cortical information processing in migraine patients between attacks and its normalization during the attack (19). In a randomized trial, 5-HTP was as effective for migraine prevention as methysergide and had fewer side effects (19), but this result is awaiting confirmation. Reduced brain serotonin synthesis might thus contribute to migraine pathogenesis, and it is well established that estrogen is able to influence enzymes at various steps of the tryptophan metabolism (21).

The aim of this study was to determine in rats if an increase of serotonin anabolism by 5-HTP administrations is able to modulate susceptibility to $\mathrm{KCL}$-induced CSD and if ovarian hormones, especially estrogen, modulate the effect.

\section{Materials and methods}

\section{Animals}

Male $(n=16)$ and female $(n=129)$ adult Sprague-Dawley rats were used in this study. They were raised and maintained under standard laboratory conditions, with tap water and regular rat chow available ad libitum on a 12 hour dark, 12 hour light cycle. All animal procedures and care complied with the guidelines of the International Association for the Study of Pain and the European Communities Council (86/609/ECC), and were approved by the institutional Ethics Committee.

\section{Procedures and experimental groups}

Cortical spreading depression (CSD). CSDs were elicited and recorded according to the method 
previously described $(8,22)$. Briefly, anesthesia was induced with chloral hydrate $(400 \mathrm{mg} / \mathrm{kg})$ and maintained by repeated smaller doses. Rectal temperature was maintained between 36.5 and $37.0^{\circ} \mathrm{C}$ using a thermostatically controlled heating blanket (ATC $1000^{\circledR}$, WPI Inc, USA). The rats were placed in a stereotactic frame (David Kopf Instruments, USA). Three 1-2mm wide burr holes were drilled 2 $\mathrm{mm}$ off the midline: $7 \mathrm{~mm}$ posterior to bregma (P-7; occipital cortex; stimulation site), $4 \mathrm{~mm}$ posterior to bregma ( $\mathrm{P}-4$; parieto-occipital recording site) and $1 \mathrm{~mm}$ anterior to bregma $(F+1$; frontal recording site).

CSDs were induced by applying a cotton ball soaked with $1 \mathrm{M} \mathrm{KC1}$ placed over the pial surface at the occipital site. CSDs were counted for 1 hour and the results were expressed as number of CSDs per hour. As proposed in other studies (23), only negative DC shifts of $\geq 5 \mathrm{mV}$ were counted as CSD. We determined the ratio between parieto-occipital and frontal CSDs and the propagation velocity of the first CSD between the parieto-occipital and frontal recording sites.

$\mathrm{DC}$ potential shifts were recorded with $\mathrm{Ag} / \mathrm{AgCl}$ wire electrodes inserted into glass micropipettes filled with artificial cerebrospinal fluid $(\mathrm{NaCl} 130 \mathrm{mM}, \mathrm{MgS0} 41.25 \mathrm{mM}, \mathrm{KC} 13.5 \mathrm{mM}, \mathrm{NaH} 2 \mathrm{P} 041.25 \mathrm{mM}$, $\mathrm{CaC} 12$ 2mM, NaHC03 $24 \mathrm{mM}$, glucose $10 \mathrm{mM}, \mathrm{pH}$ 7.4). The electrodes were lowered into the cortex (average depth: $800 \mu \mathrm{m}$ below the dural surface). An $\mathrm{Ag} / \mathrm{AgCl}$ reference electrode was inserted subcutane-ously in the neck. The electrical signals were amplified with an ISODAM-8A bioamplifier at a DC-10kHz bandwidth (WPI Inc, USA), digitized at a $200 \mathrm{~Hz}$ sampling rate and stored for off-line analysis using Microl401 MKII and Spike2 software (CED Co., UK).

5-hydroxytryptophan (5-HTP) administration. One hour before the CSD recordings, the animals received an intraperitoneal (i.p.) injection of physiological saline ( $\mathrm{NaCl}$ groups) or of 5-HTP (5-HTP groups). We chose a $100 \mathrm{mg} / \mathrm{kg}$ dose of 5-HTP on the basis of a number of literature data showing that this dose is highly effective and well tolerated in various biochemical $(24,25,26)$ or behavioral studies $(27,28)$. The one hour delay between $5-\mathrm{HTP}$ injections and CSD recordings is the delay at which 5-HT brain levels peak in studies of various animal species including rats $(24,29)$. 5-hydroxytryptophan (5HTP) was obtained from Sigma (Steinheim, Germany).

\section{Experimental groups}

Females compared to males. We first studied the effect of 5-HTP administrations on CSD in a group of 32 animals, 16 females (estrous cycle phase not determined) and 16 males. In each subgroup, eight animals received an i.p. injection of physiological saline, and eight others received $100 \mathrm{mg} / \mathrm{kg} 5-\mathrm{HTP}$.

Female subgroups according to estrous cycle phase. Given the results of the first experiment, another group of 48 female rats was used in order to assess the influence of hormone variations during the estrous cycle on CSD frequency and its modulation by 5-HTP. Three phases were analysed: Proestrus, estrus and diestrus ( $n=16$ per phase). The latter includes metestrus, the two being also called diestrus-1 and diestrus-2 (30). Vaginal smears were taken under anesthesia between $10 \mathrm{am}$ and $2 \mathrm{pm}$ just before the electrophysiological recordings and colored with the Giemsa stain to determine the phase of the cycle.

Oophorectomy and hormonal treatments. Sixty-five female rats were bilaterally oophorectomized under isoflur-ane inhalation $\left(2\right.$ to $3 \%$ in a flow of $1 \mathrm{~L} / \mathrm{min}$ of oxygen, Forene ${ }^{\circledR}$, Abbott, Queenborough, Kent, England) and subcutaneously implanted with $1-\mathrm{cm}$ long silastic capsules. Estrogen implants were filled with a $20 \% 17 \beta$-estradiol-cholesterol mixture $(E 2, n=32)$, whereas control implants were $100 \%$ cholesterol filled (Choi, $n=33$ ). Implants of such length and concentration are known to reproduce the physiological peak of proestrus blood levels of hormone (31). Two weeks later, CSDs were elicited in half of the animals after i.p. injections of 5-HTP $(E 2, n=7$; Chol, $n=8)$ or of $\mathrm{NaCl}(E 2$, $\mathrm{n}=9$; Chol, $\mathrm{n}=8$ ), while in the remaining animals the capsules were removed to reproduce a fall of $E 2$ and CSDs were recorded $20 \mathrm{~h}$ later after 5-HTP (E2w, $\mathrm{n}=8$; Cholw, $\mathrm{n}=8$ ) or $\mathrm{NaCl}(\mathrm{E} 2 \mathrm{w}, \mathrm{n}=8$; Cholw, $\mathrm{n}=9$ ) administration. 17ß-estradiol (E2) and cholesterol (Chol) were obtained from Sigma (Steinheim, Germany).

\section{Statistical analyses}

Group values were expressed as means \pm standard error of means. To evaluate the effect of 5-HTP 
administration and sex on hourly CSD frequency and propagation velocity of the first CSD, we used two factor ANOVAs [treatment $(\mathrm{NaCl} / 5-\mathrm{HTP}) \times \operatorname{sex}(\mathrm{male} / \mathrm{female})]$. To evaluate the effect of the estrous cycle phases and hormonal treatments after oophorectomy on 5-HTP-induced CSD changes, a two factor ANOVA ([treatment $(\mathrm{NaCl} / 5-\mathrm{HTP}) \times$ cycle (diestrus/ proestrus/estrus)] and [treatment $(\mathrm{NaCl} / 5-\mathrm{HTP}) \times$ hormone (Chol/Chow/E2/E2w)]) was also applied followed by Duncan's post-hoc test. Analyses were implemented in SAS version 9.2 (SAS institute, Cary, NC, USA) with $p<0.05$ as threshold for statistical significance.

\section{Results}

\section{5-HTP effects on CSD}

In the first group of 32 animals, intraperitoneal injections of 5-HTP numerically decreased CSD frequency at the parieto-occipital recording site (Figure 1). This decrease, however, only reached a statistical trend when data from both sexes $(n=16)$ were pooled (ANOVA, treatment factor, $F(1,28)=$ $3.449, p=0.073$ ) The sex of the animals had no significant influence on the effect of 5-HTP on CSD (ANOVA, sex factor, $\mathrm{F}(1,28)=1.653, p=0.20$ ), but in the subgroup of $\mathrm{NaCl}$-injected animals, hourly CSD frequency tended to be greater in females than in males $(p=0.08)$.

At the frontal recording site, we found no significant difference in CSD frequency between males and females, or between $\mathrm{NaCl}$ and 5-HTP treated animals, although the latter had numerically fewer CSDs (Figure 1). Similarly for propagation velocity, there was no significant difference between $\mathrm{NaCl}$ - and 5HTP-treated males $(3.63 \pm 0.12$ and $3.50 \pm 0.12 \mathrm{~mm} / \mathrm{min})$, or between the two female treatment groups $(3.35 \pm 0.18$ and $3.09 \pm 0.10 \mathrm{~mm} / \mathrm{min})$.

\section{Estrous cycle and 5-HTP effects on CSD}

In the group of 48 female animals in which the phases of the estrous cycle were determined, there was a significant change in parieto-occipital CSD frequency over the estrous cycle (ANOVA cycle factor, $F$ $(2,42)=8.343, p<0.001)$ (Figure 2). After analysis of parieto-occipital CSD frequency by one-way ANOVA followed by post-hoc Duncan's test, the CSD frequency was lowest during estrus compared to proestrus (Duncan's test, estrus vs proestrus, $p<0.001$ ) or diestrus (Duncan's test, estrus vs diestrus, $p=0.037$ ). There was no significant difference between proestrus and diestrus (Duncan's test, proestrus vs diestrus, $p=0.15$ ).

In the total group of 48 females, 5-HTP significantly decreased CSD occurrence at the parieto-occipital recording site (ANOVA, treatment factor, $F(1,42)=11.945, p=0.001$ ). When subanalyzed in each phase of the ovarian cycle, 5-HTP significantly reduced CSD frequency only during estrus (Duncan's test, $\mathrm{NaCl}$ estrus vs 5 -HTP estrus, $\mathrm{p}<0.001$ ) but not during diestrus (Duncan's test, $\mathrm{NaCl}$ diestrus vs 5 -HTP diestrus, $p=0.18$ ) or proestrus (Duncan' test, NaCl proestrus vs 5 -HTP proestrus, $p$ $=0.30$ ) (Figure 2).

At the frontal recording site the results were similar but not all were significant. The estrous cycle tended to influence frontal CSD frequency (ANOVA, cycle factor, $F(1,42)=2.721, p=0.077$ ). 5-HTP administration significantly decreased frontal CSD frequency (ANOVA, treatment factor, $F(1,42)$ $=6.517, p=0.014$ ), particularly during the estrus phase (Duncan's test, $5-\mathrm{HTP}$ vs $\mathrm{NaCl}, p=0.017$ ) (Table 1).

The velocity of the first CSD propagated between parieto-occipital and frontal recording sites was not significantly influenced by 5 -HTP treatment (ANOVA, treatment factor, $\mathrm{F}(1,42)=0.781, p=0.38$ ) nor by the estrous cycle (ANOVA, cycle factor, $\mathrm{F}(2,42)=0.260, p=0.38$ ) (Table 1 ). 
Figure 1. Number of CSD/hour (mean \pm sem) at the occipito-parietal and frontal recording sites after intraperitoneal injection of $\mathrm{NaCl}$ or 5 -HTP in male and female rats $\left(\mathrm{n}=8\right.$ per group). ${ }^{(*)} p=0.08$ females- $\mathrm{NaCl}$ vs. males-NaCl).

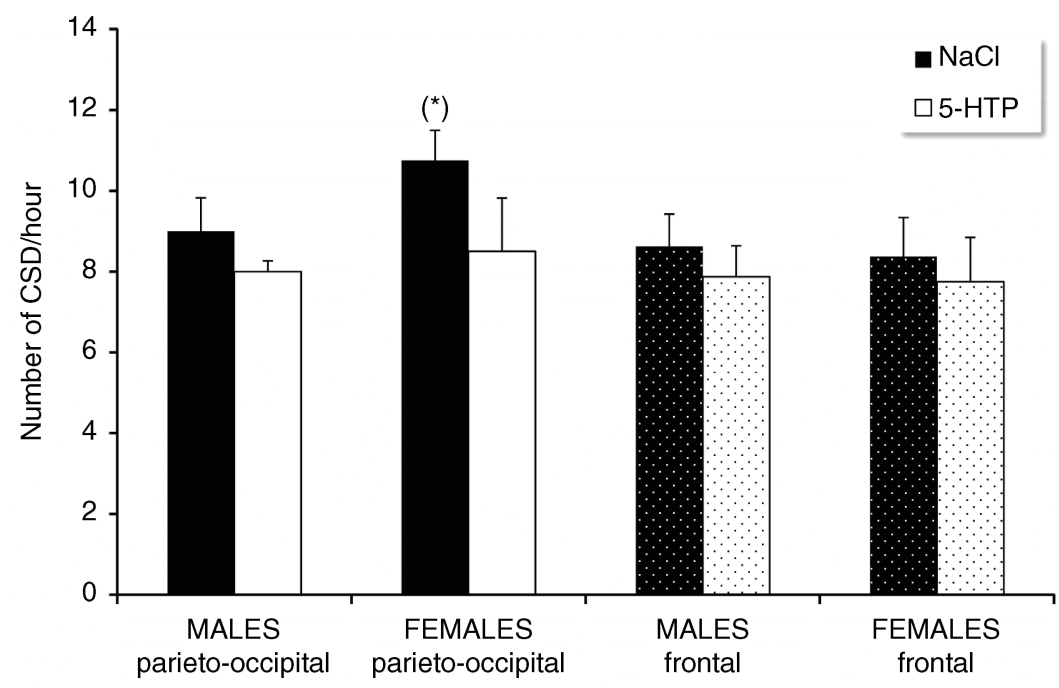

Figure 2. Number of CSD/hour at the occipito-parietal recording site in female rats after i.p. injection of 5 -HTP or $\mathrm{NaCl}$ during three phases of the estrous cycle determined by vaginal smears $\left({ }^{* \star} p<0.001,5-\right.$ HTP vs. $\mathrm{NaCl}$ treatment; ${ }^{\#} p<0.05$ diestrus vs. estrus; ${ }^{\# \#} p<0.001$ proestrus vs. estrus) ( $n=8$ per group).

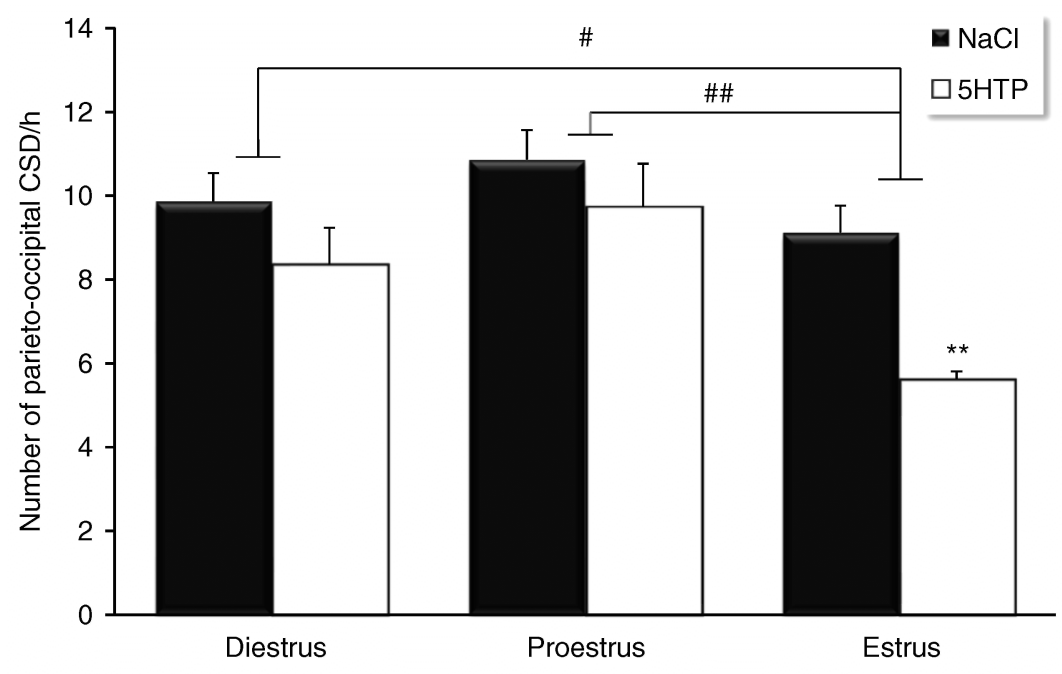


Figure 3. Number of $\mathrm{CSD} /$ hour at the parieto-occipital recording site in oophorectomized female rats implanted for two weeks with cholesterol (Chol)- or 17ß-estradiol (E2)-filled silastic capsules and one day after capsule withdrawal (Cholw and E2w). Effects of i.p. injection of $5-\mathrm{HTP}$ or $\mathrm{NaCl}\left({ }^{* *} p<0.01\right.$ $\mathrm{NaCl}$ E2 vs. 5 -HTP E2; ${ }^{\# \#} p<0.001 \mathrm{NaCl}$ E2 vs. $\left.\mathrm{NaCl} \mathrm{Chol}\right)$.

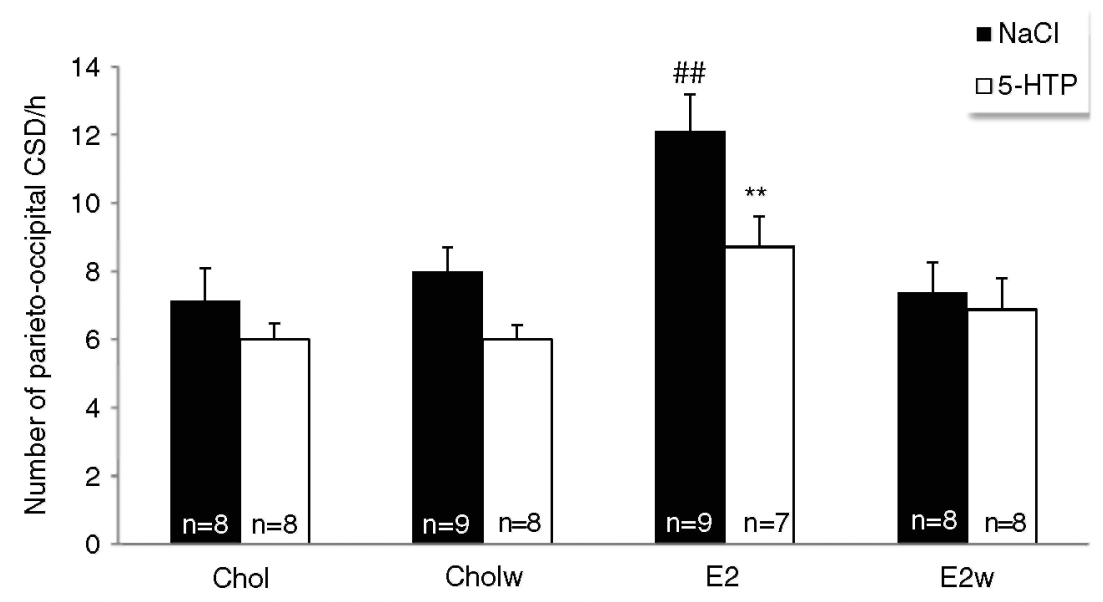

Table 1. CSD frequencies measured at the frontal recording site and propagation velocity of the first CSD after i.p. injection of $\mathrm{NaCl}$ or 5-HTP compared between the three phases of the estrous cycle determined by vaginal smears (means \pm sem) $\left({ }^{*} p<0.015-\mathrm{HTP}\right.$ vs. $\mathrm{NaCl}$ treatment) ( $\mathrm{n}=8$ per group).

\begin{tabular}{lcc}
\hline \multicolumn{1}{c}{$\mathrm{NaCl}$} & $5-\mathrm{HTP}$ \\
\hline CSD frequency at frontal recording site & \\
Diestrus & $8.75 \pm 0.77$ & $7.63 \pm 0.84$ \\
Proestrus & $8.38 \pm 0,26$ & $7.50 \pm 1.07$ \\
Estrus & $7.88 \pm 0,74$ & $5.38 \pm 0.18^{\star}$ \\
CSD propagation velocity $(\mathrm{mm} / \mathrm{min})$ & \\
Diestrus & $3.42 \pm 0.19$ & $3.25 \pm 0.29$ \\
Proestrus & $3.28 \pm 0.16$ & $3.02 \pm 0.09$ \\
Estrus & $3.00 \pm 0.19$ & $3.10 \pm 0.12$
\end{tabular}

\section{Estrogen replacement therapy and 5-HTP effects on CSD}

In the 65 oophorectomized female animals taken together, estradiol replacement therapy significantly influenced parieto-occipital CSD frequency (ANOVA, hormonal factor, $F(3,61)=8.835, p<0.001$ ) (Figure 3). In rats receiving $\mathrm{NaCl}, \mathrm{CSD}$ frequency was markedly increased after implantation with E2filled capsules compared to cholesterol-filled capsules (Figure 3). (Duncan's test, $\mathrm{NaCl} \mathrm{E2} \mathrm{vs.} \mathrm{NaCl}$ Chol, $p<0.001$ ). When animals injected with $\mathrm{NaCl}$ or 5 - HTP were analyzed separately, the CSDenhancing effect of $\mathrm{E} 2$ was significant only after $\mathrm{NaCl}$ (Duncan's test, $\mathrm{NaCl} \mathrm{E} 2$ vs $\mathrm{NaCl}$ Chol, $p<$ $0.001 ; \mathrm{NaCl} E 2$ vs. NaCl Cholw, $p=0.001 ; \mathrm{NaCl}$ E2 vs. NaCl E2w, $p<0.001$ ), but not after $5-\mathrm{HTP}$ (Figure 3).

Globally, 5-HTP administration significantly decreased CSD occurrence at the parieto-occipital recording site (ANOVA, treatment factor, $F(1,57)=8.872, p=0.004$ ). When analyzed in detail, this effect reached the level of significance only in E2-treated animals (Duncan's test, 5 -HTP E2 vs NaCl E2, $p=0.006$ ) (Figure 3).

Similar changes were observed at the frontal level, where estrogen replacement treatment also increased CSD frequency (ANOVA, hormone factor, $F(3,61)=2.826, p=0.046$ ). The effect of E2 was, however, less consistent than for parieto-occipital CSD. When $\mathrm{NaCl}$-injected oophorectomized females were considered, there was a significant increase of CSD frequency in the E2 group compared to the Chol group ( $\mathrm{NaCl} \mathrm{E} 2$ vs. $\mathrm{NaCl} \mathrm{Chol}, p=0.008$ ), but no more than a trend compared to $\mathrm{E} 2$ withdrawal $(\mathrm{NaCl} \mathrm{E} 2$ vs. $\mathrm{NaCl} \mathrm{E} 2 \mathrm{w}, p=0.094)$ and no significant difference compared to 
cholesterol withdrawal ( $\mathrm{NaCl}$ E2 vs. $\mathrm{NaCl}$ Cholw, $p=0.50$ ) (Table 2).

Overall, 5-HTP treatment significantly decreased frontal CSD frequency (ANOVA, treatment factor,

$F(1,63)=4.705, p=0.034)$, but this was mainly due to the CSD-inhibitory effect in E2-treated animals (Duncan's test, NaCl E2 vs 5-HTP E2, $p=0.029$ ) (Table 2).

The velocity of the first CSD propagated between parieto-occipital and frontal levels was not significantly influenced by hormonal treatment (ANOVA, hormone factor, $F(3,61)=1.439, p=0.24$ ), nor by 5 -HTP injections (ANOVA, treatment factor, $\mathrm{F}(3,62)=2.215, p=0.14)$ (Table 2).

Table 2. CSD frequencies measured at the frontal recording site and propagation velocities of the first CSD after i.p. injection of $\mathrm{NaCl}$ or 5-HTP compared in oophorectomized female rats treated for two weeks with cholesterol (Chol)- or 17ß-estradiol (E2)-filled silastic capsules (Chol and E2) and after capsule withdrawal (Cholw and E2w), (means \pm sem) $\left({ }^{*} p<0.01 \mathrm{NaCl}\right.$ E2 vs. NaCl Chol; ${ }^{\#} p=0.029$ $\mathrm{NaCl}$ E2 vs. 5-HTP E2).

$$
\mathrm{NaCl} 5 \text {-HTP }
$$

CSD frequency at frontal recording site

$\begin{array}{lll}\text { Chol } & 5.25 \pm 0.31 & 6.13 \pm 0.48 \\ \text { Cholw } & 6.00 \pm 0.50 & \star 5.88 \pm 0.74 \\ \text { E2 } & 8.33 \pm 0.53- & 6.57 \pm 0.81^{\#} \\ \text { E2w } & 6.63 \pm 1.32 & 6.25 \pm 0.88\end{array}$

CSD propagation velocity $(\mathrm{mm} / \mathrm{min})$

$\begin{array}{lll}\text { Chol } & 3.34 \pm 0.27 & 3.36 \pm 0.50 \\ \text { Cholw } & 3.35 \pm 0.17 & 3.83 \pm 0.47 \\ \text { E2 } & 3.23 \pm 0.10 & 3.03 \pm 0.23 \\ \text { E2w } & 3.44 \pm 0.33 & 4.06 \pm 0.42\end{array}$

\section{Discussion}

Serotonin, cortical excitability and sex hormones all play a role in migraine pathogenesis. Our purpose was to explore the possible interrelation between these factors in rats. We confirm that estrogens increase cortical excitability as indexed by hourly frequency of $\mathrm{KCl}$-induced cortical spreading depressions (CSD). We find that intraperitoneal administration of 5-hydroxytryptophan (5-HTP), deemed to increase serotonin levels in the brain, decreases cortical excitability and that this effect depends on the estrogen status of the animal.

Increasing $5-\mathrm{HT}$ disposition by $5-\mathrm{HT}$ reuptake inhibitors like d-fenfluramine in male rats (32) or fluoxetine in pups and female rats (33) and by acute administrations of tryptophan (34) was reported to reduce CSD propagation velocity. We aimed at increasing brain 5-HT levels by systemic injections of 5-HTP in order to bypass tryptophan hydroxylase, the rate-limiting enzyme in serotonin synthesis. 5HTP failed to significantly decrease CSD frequency or propagation velocity in male rats. In females, taken as a group irrespective of their hormonal status, there was a numerical decrease in hourly CSD frequency that did not reach the level of significance, probably because of the large inter-subject variability. However, when the number of female animals was increased in the second experiment, it became obvious that globally the 5-HTP injection significantly decreased CSD frequency and that this was chiefly due to an inhibitory effect during the estrus phase of the ovarian cycle.

Ovarian sex hormones thus seem to be necessary to unravel the CSD-suppressive effect of 5-HTP. While 5-HTP has no significant effect on CSD frequency in males, it decreases this frequency in females. This could be partly favored by the fact that intact female rats have on average a higher baseline CSD frequency during saline injections than males, in particular during proestrus when estrogen levels peak. Estrogen is able per se to increase CSD susceptibility, as confirmed in our study. We found that after oophorectomy CSD frequency is markedly increased in animals treated with estradiol compared to controls without replacement therapy. We show, in addition, that the effect of estrogen on CSD in oophorectomized animals is short-lasting: The day following removal of the implanted capsule containing estradiol, CSD frequency had dropped to the level found in rats implanted with cholesterol-filled capsules. The sensitizing effect of estrogen on CSD susceptibility was 
reported in the knock-in mouse model of familial hemiplegic migraine type 1 (10), but in this study there was no sex difference in wild type mice. We have previously described the CSD sensitizing effect of estrogen in normal and E2-treated oophorectomized rats (9). Estrogen can modify susceptibility to CSD by several mechanisms, but mainly through its effect on glutamate neurotransmis-sion, which is known to promote CSD generation. Estrogen affects neuronal plasticity during the estrous cycle by increasing the number of dendritic spines, and of synaptic densities via an NMDA receptor dependent mechanism $(35,36)$. It up-regulates NMDA receptors and down-regulates glutamate uptake by astrocytes (37). All these effects of estrogen should enhance glutamate transmission and neuronal excitability, facilitating the occurrence of CSD.

The 5-HTP effect is not the only CSD-suppressing effect that clearly depends on the estrogen status of the animal. In a previous study, we found similar estrogen dependence for the inhibitory effect of Lkynurenine on CSD susceptibility (9). Over the rat estrous cycle, estrogen peaks during the day of proestrus, while progesterone does so during the night between proestrus and estrus. During estrus, the plasma levels of both hormones are low (30). The decrease in CSD frequency during estrus could be due to the fall in estrogen during this phase. However, estrogen has genomic and non-genomic actions that may determine respectively long and short term effects. Since the ovarian cycle of rats is short (four or five days), some long-term effects of estrogen may persist on the day following proestrus, i.e., during estrus. To solve this question, we have assessed the effect of 5-HTP on CSD frequency in oophorectomized rats chronically treated, or not, with estradiol, and after its withdrawal. The inhibitory effect of 5-HTP on CSD frequency is evident in females chronically treated with estradiol after oophorectomy, but disappears the day following estradiol withdrawal. This is at variance with the changes found during spontaneous estrous cycles, where the CSD-suppressing effect of 5-HTP is significant during estrus, i.e., when estrogen blood levels are low. If the CSD suppressing effect of 5HTP in cycling females during estrus were due to a long-term effect of estrogens, it could have persisted the day after estradiol withdrawal, which was not the case. For obvious reasons, however, the results obtained in oophorectomized females treated with estradiol for two weeks cannot be translated without reservation to those found in spontaneously cycling females in whom the high estrogen level during proestrus lasts for no more than 24 hours. Given these results, and the fact that progesterone, whose blood levels peak between proestrus and estrus, can also modify cortical excitability, our study does not allow disentanglement of the precise hormonal underpinning of the observed CSD suppressive effect of 5-HTP.

Many studies have investigated the effect of ovarian steroids on the serotoninergic system. Their action varies between cerebral areas and neuronal circuits (38), and contradictory results have been reported. Estrogen can exert direct and indirect effects on serotonin transmission, and is able to modulate the density and function of the $5-\mathrm{HTT}, 5-\mathrm{HT}_{1 \mathrm{~A}}$ and $5-\mathrm{HT}_{2 \mathrm{~A}}$ receptors as well as $5-\mathrm{HT}$ synthesis and degradation (38). Autoradiographic studies with specific ligands or in situ mRNA hybridization have shown in the cortex, hippocampus and raphe a decrease of $5-\mathrm{HT}_{1 \mathrm{~A}}$ receptors or no effect after short or long term estrogen treatment in oophorectomized rats $(39,40)$. Estrogen was shown to decrease raphe $5-\mathrm{HT}_{1 \mathrm{~A}}$ somato-dendritic receptors, but to increase postsynaptic receptors in the cortex (41). The affinity of cortical serotonergic receptors is weak during proestrus, but increases during estrus, and this change is more pronounced for $5-\mathrm{HT}_{1}$ than for $5-\mathrm{HT}_{2}$ receptors (41). Estrogen negatively regulates $5-\mathrm{HT}_{1 \mathrm{~B}}$ autoreceptor mRNA in raphe nuclei (42) and increases $5-\mathrm{HT}_{2 \mathrm{~A}}$ receptors in several cerebral regions, including raphe nuclei and the frontal cortex (43). With respect to our experiments, one must add that there may be, in addition, gender differences in the transformation of 5-HTP into 5-HT. Literature data, however, are scarce and partly contradictory.

In the mediobasal hypothalamus of neonatal rats, neurons immunoreactive for amino-acid decarboxylase (AADC), the enzyme transforming 5-HTP into 5-HT, are more numerous in females than in males (44). By contrast, in the trigeminal ganglion of mice, AADC mRNA is more expressed in males than females, and in females much more in proestrus than in estrus (45). Following AADC inhibition, 5-HTP accumulation in the brain was more pronounced in females than in males (46), but there was no effect of sex in a PET study assessing AADC in rhesus monkey brains (47). Finally, in human studies, the effect of 5-HTP on cho-lecystokinin-4-induced panic attacks (48) or hormonal responses $(49,50)$ was more obvious in females than in males. Taken together, these studies support the notion that overall estrogen increases sensitivity and activity of the central serotoninergic system, and possibly the transformation of 5-HTP into 5-HT. Transposed to our study, this may explain why estrogen tends to unravel or amplify the CSD-suppressive effect of 5-HTP administrations. 
Although the findings presented here cannot be translated without reservation to clinical migraine, they may shed light on clinical features related to hormonal fluctuations and encourage revisiting poorly explored therapeutic strategies. The CSD-sensitizing effect of estrogen can explain why migraine with aura attacks are favored by pregnancy (11) and treatment with estrogen-containing drugs (12), but ameliorated by substituting an estro-progestative contraceptive pill by a desogestrel only-containing contraceptive (51). One must keep in mind, however, that the E2 levels in pregnancy are much higher than those found in proestrus or during post-oophorectomy replacement treatment. By contrast, the vast majority of menstrual-related migraine attacks are without aura and supposed to be triggered by the premenstrual fall in plasma estrogen levels (52). This is in line with our finding in oophorectomized rats, showing that the increased CSD susceptibility induced by estradiol treatment has disappeared one day after treatment arrest. By corollary, this would suggest that most menstrual-related migraine attacks are not caused by CSD, and hence that attacks of migraine without aura are not associated with CSD.

The CSD-suppressive effect of 5-HTP found in our study may indicate that 5-HTP merits further attention in migraine therapy, in addition to the sole available small trial showing that it was as effective for migraine prevention as methysergide (20). The experimental data would suggest that 5HTP might be effective in migraine with aura, more so in females than in males, and more so in females with high estrogen blood levels. This could be taken into consideration for the design of future therapeutic protocols.

To conclude, the serotoninergic system and ovarian hormones play a complex and interconnected role in migraine pathogenesis. Increase of 5-HT levels by administering 5-HTP decreases CSD susceptibility in female rats. This effect is more pronounced during the estrus phase in cycling rats and during estradiol treatment in oophorectomized rats, which per se increases CSD susceptibility. It is short-lived, as it disappears within 24 hours after estradiol withdrawal, and could be explained by a sensitizing effect of estrogen on serotonin neurotransmission. Transposed to the clinic, these findings may explain why aura attacks are favored by high estrogen levels, as during pregnancy or intake of an estrogen-progesterone contraceptive pill, and why the majority of menstrual-related migraine attacks due to the fall of estrogen plasma levels are without aura. Finally, because of its CSD-suppressive effect, 5-HTP might merit further therapeutic trials, particularly in women suffering from migraine with aura and with elevated estrogen levels.

\section{Article highlights}

- Increasing 5-HT disposition by administration of 5-HTP decreases $\mathrm{KCl}$-induced CSD frequency in female rats.

- The CSD suppressive effect of 5-HTP only appears in the presence of ovarian sex hormones. It is more pronounced during the estrous phase in cycling rats and after chronic E2 administration in oophorectomized animals.

- Elevated E2 levels increase CSD frequency, while E2 withdrawal promptly normalizes it.

- Transposed to the clinic, these results suggest that 5-HTP may have therapeutic potentials for migraine with aura in females. They also may explain why migraine with aura can be aggravated/triggered by high E2 states, and why menstrual-related attacks are rarely associated with an aura.

\section{Ethics or Institutional Review Board approval}

All procedures and care complied with the guidelines of the International Association for the Study of Pain and the European Communities Council (86/609/ECC) and were approved by the Ethics Committee for animal experiments of the University of Liège-Belgium (Approval LA1610002).

\section{Acknowledgements}

The authors thank Jeanine Mosen, Alexandra Pieltain and Dr Vladimir Bogdanov for their assistance and advice during the electrophysiological studies, and Laurence Seidel from the Department of Biomedical Statistics of the University of Liege for the statistical analyses. 


\section{Declaration of conflicting interests}

The authors declared no potential conflicts of interest with respect to the research, authorship, and/or publication of this article.

\section{Funding}

The authors disclosed receipt of the following financial support for the research, authorship, and/or publication of this article: The Belgian National Fund for Scientific Research (FNRS) [convention 3.4.650.09] and Special Research Funds of the University of Liège supported his work and partly FP7EUROHEADPAIN no. 602633.

\section{References}

1. Marcus DA. Interrelationships of neurochemicals, estrogen, and recurring headache. Pain 1995; 62: 129-139.

2. Lauritzen M. Pathophysiology of the migraine aura. The spreading depression theory. Brain 1994; 117: 199-210.

3. Leao A. Spreading depression of activity in cerebral cortex. J Neurophysiol 1944; 7: 159-390.

4. Zhang $X$, Levy $D$, Kainz $V$, et al. Activation of central trigeminovascular neurons by cortical spreading depression. Ann Neurol 2011; 69: 855-865.

5. Goadsby PJ. Migraine, aura, and cortical spreading depression: Why are we still talking about it? Ann Neurol 2001; 49: 4-6.

6. Brennan KC, Romero Reyes M, Lopez Valdes HE, et al. Reduced threshold for cortical spreading depression in female mice. Ann Neurol 2007; 61: 603-606.

7. Sachs M, Pape HC, Speckmann EJ, et al. The effect of estrogen and progesterone on spreading depression in rat neocortical tissues. Neurobiol Dis 2007; 25: 27-34.

8. Chauvel V, Vamos E, Pardutz A, et al. Effect of systemic kynurenine on cortical spreading depression and its modulation by sex hormones in rat. Exp Neurol 2012; 236: 207-214.

9. Chauvel V, Schoenen $\mathrm{J}$ and Multon S. Influence of ovarian hormones on cortical spreading depression and its suppression by L-kynurenine in rat. PLoS One 2013; 10: 8-12.

10. Eikermann-Haerter K, Dilekoz E, Kudo C, et al. Genetic and hormonal factors modulate spreading depression and transient hemiparesis in mouse models of familial hemi-plegic migraine type 1. J Clin Invest 2009; 119: 99-109.

11. Maggioni F, Alessi C, Maggino T, et al. Headache during pregnancy. Cephalalgia 1997; 17: 765769.

12. Granella F, Sances G, Pucci E, et al. Migraine with aura and reproductive life events: A case control study. Cephalalgia 2000; 20: 701-707. 
13. Schoenen J. Migraine and serotonin: The quest for the Holy Grail goes on. Cephalalgia 2014; 3: 163-164.

14. Ferrari MD and Saxena PR. On serotonin and migraine: A clinical and pharmacological review. Cephalalgia 1993; 13: 151-165.

15. Sicuteri F, Testi A and Anselmi B. Biochemical investigations in headache: Increase in the hydroxyindoleacetic acid excretion during migraine attacks. Int J Allergy Immunol 1961; 19: 55-58.

16. Humphrey PP. 5-Hydroxytryptamine and the patho-physiology of migraine. J Neurol 1991; 238: S38-44.

17. Sakai $Y$, Dobson $C$, Diksic $M$, et al. Sumatriptan normalizes the migraine attack-related increase in brain serotonin synthesis. Neurology 2008; 6: 431-439.

18. Supornsilpchai W, Sanguanrangsirikul S, Maneesri S, et al. Serotonin depletion, cortical spreading depression, and trigeminal nociception. Headache 2006; 46: 34-39.

19. Judit A, Sandor PS and Schoenen J. Habituation of visual and intensity dependence of auditory evoked cortical potentials tends to normalize just before and during the migraine attack. Cephalalgia 2000; 20: 714-719.

20. Titus F, Davalos A, Alom J, et al. 5-Hydroxytryptophan versus methysergide in the prophylaxis of migraine. Randomized clinical trial. Eur Neurol 1986; 25: 327-329.

21. Donner $\mathrm{N}$ and Handa R. Estrogen receptor beta regulates the expression of tryptophanhydroxylase 2 mRNA within serotonergic neurons of the rat dorsal raphe nuclei. Neuroscience 2009; 163: 705-718.

22. Bogdanov VB, Multon $\mathrm{S}$, Chauvel V, et al. Migraine preventive drugs differentially affect cortical spreading depression in rat. Neurobiol Dis 2011; 41: 430-435.

23. Ayata $\mathrm{C}$, Jin $\mathrm{H}$, Kudo $\mathrm{C}$, et al. Suppression of cortical spreading depression in migraine prophylaxis. Ann Neurol 2006; 59: 652-661.

24. Gartside SE, Cowen PJ and Sharp T. Effect of 5-hydroxy-L-tryptophan on the release of 5-HT in rat hypothalamus in vivo as measured by microdialysis. Neuropharmacology 1992; 31: 9-14.

25. Choi SH, Kwon BS, Lee S, et al. Systemic 5-hydroxy-L-tryptophan down-regulates the arcuate CART mRNA level in rats. Regul Pept 2003; 115: 73-80.

26. Fox MA, Jensen CL, French HT, et al. Neurochemical, behavioral and physiological effects of pharmacologically enhanced serotonin levels in serotonin transporter (SERT)-deficient mice. Psychopharmacology (Berl) 2008; 201: 203-218.

27. Amer A, Breu J, McDermott J, et al. 5-Hydroxy-L-tryp-tophan suppresses food intake in fooddeprived and stressed rats. Pharmacol Biochem Behav 2004; 77: 137-143.

28. Turner EH, Loftis JM and Blackwell AD. Serotonin a la carte: Supplementation with the serotonin precursor 5-hydroxytryptophan. Pharmacol Ther 2006; 109: 325-338.

29. Das YT, Bagchi M, Bagchi D, et al. Safety of 5-hydroxy-L-tryptophan. Toxicol Lett 2004; 150: 111122.

30. Freeman ME. Neuroendocrine control of the ovarian cycle of the rat. In: Neill JD (eds) Knobil and Neill's Physiology of Reproduction. Elsevier Academic Press, 2006, pp. 2327-2388.

31. Gogos A and Van den Buuse M. Estrogen and progesterone prevent disruption of prepulse inhibition by the

serotonin-1A receptor agonist 8-hydroxy-2-dipropylami-notetralin. J Pharmacol Exp Ther 2004; 309: 267-274.

32. Cabral-Filho JE, Trindade-Filho EM and Guedes RC. Effect of d-fenfluramine on cortical spreading depression in rats. Braz J Med Biol Res 1995; 28: 347-350.

33. dos Santos AA, Pinheiro PC, de Lima DS, et al. Fluox-etine inhibits cortical spreading depression in weaned and adult rats suckled under favorable and unfavorable lactation conditions. Exp Neurol 
2006; 200: 275-282.

34. Trindade-Filho EM, de Vasconcelos CAC and Guedes RCA. Acute tryptophan administration impairs cortical spreading depression propagation in REM sleep deprived and nondeprived adult rats. Psych Neurosci 2009; 2: 235-241.

35. Brinton RD, Proffitt P, Tran J, et al. Equilin, a principal component of the estrogen replacement therapy pre-marin, increases the growth of cortical neurons via an NMDA receptor-dependent mechanism. Exp Neurol 1997; 147: 211-220.

36. Woolley CS and McEwen BS. Estradiol mediates fluctuation in hippocampal synapse density during the estrous cycle in the adult rat. $J$ Neurosci 1992; 12: 2549-2554.

37. Sato K, Matsuki N, Ohno Y, et al. Estrogens inhibit 1-glutamate uptake activity of astrocytes via membrane estrogen receptor alpha. J Neurochem 2003; 86: 1498-1505.

38. Bethea CL, Lu NZ, Gundlah C, et al. Diverse actions of ovarian steroids in the serotonin neural system. Front Neuroendocrinol 2002; 23: 41-100.

39. Osterlund MK and Hurd YL. Acute 17 beta-estradiol treatment down-regulates serotonin $5 \mathrm{HT} 1 \mathrm{~A}$ receptor mRNA expression in the limbic system of female rats. Brain Res Mol Brain Res 1998; 55: 169-172.

40. Le Saux M and Di Paolo T. Changes in 5-HT1A receptor binding and G-protein activation in the rat brain after estrogen treatment: Comparison with tamoxifen and raloxifene. J Psychiatry Neurosci 2005; 30: 110-117.

41. Uphouse L, Williams $\mathrm{J}$, Eckols $\mathrm{K}$, et al. Variations in binding of $[3 \mathrm{H}] 5-\mathrm{HT}$ to cortical membranes during the female rat estrous cycle. Brain Res 1986; 381: 376-381.

42. Hiroi R and Neumaier JF. Estrogen decreases 5-HT1B autoreceptor mRNA in selective subregion of rat dorsal raphe nucleus: Inverse association between gene expression and anxiety behavior in the open field. Neuroscience 2009; 158: 456-464.

43. Cyr M, Bosse R and Di Paolo T. Gonadal hormones modulate 5-hydroxytryptamine2A receptors: Emphasis on the rat frontal cortex. Neuroscience 1998; 83: 829-836.

44. Balan IS, Ugrumov MV, Calas A, et al. Tyrosine hydro-xylase-expressing and/or aromatic L-amino acid decar-boxylase-expressing neurons in the mediobasal hypothalamus of perinatal rats: Differentiation and sexual dimorphism. J Comp Neurol 2000; 425: 167-176.

45. Asghari R, Lung MS, Pilowsky PM, et al. Sex differences in the expression of serotoninsynthesizing enzymes in mouse trigeminal ganglia. Neuroscience 2011; 199: 429-437.

46. Carlsson $M$ and Carlsson A. A regional study of sex differences in rat brain serotonin. Prog Neuropsychopharmacol Biol Psychiatry 1988; 12: 53-61. 
47. Eberling JL, Roberts JA, Taylor SE, et al. No effect of age and estrogen on aromatic L- amino acid decarboxyl-ase activity in rhesus monkey brain. Neurobiol Aging 2002; 23: 479-483.

48. Maron E, Tõru I, Vasar V, et al. The effect of 5-hydroxytryptophan on cholecystokinin-4-induced panic attacks in healthy volunteers. J Psychopharmacol 2004; 18: 194-199.

49. Mashchak CA, Kletzky OA, Spencer C, et al. Transient effect of L-5-hydroxytryptophan on pituitary function in men and women. J Clin Endocrinol Metab 1983; 56: 170-176.

50. Maes M, De Ruyter M, Claes R, et al. The cortisol responses to 5-hydroxytryptophan, orally, in depressive inpatients. J Affect Disord 1987; 13: 23-30.

51. Nappi RE, Sances G, Allais G, et al. Effects of an estrogen-free, desogestrel-containing oral contraceptive in women with migraine with aura: A prospective diary-based pilot study. Contraception 2011; 8: 223-228.

52. MacGregor EA. Perimenstrual headaches: Unmet needs. Curr Pain Headache Rep 2008; 12 : 468-474. 